\title{
Flame treatment of low-density polyethylene: Surface chemistry across the length scales
}

\author{
Jing Song ${ }^{\mathrm{a}}$, Ullrich Gunst ${ }^{\mathrm{b}}$, Heinrich F. Arlinghaus ${ }^{\mathrm{b}}$, G. Julius Vancso ${ }^{\mathrm{a}, *}$ \\ ${ }^{a}$ Dutch Polymer Institute and MESA ${ }^{+}$Institute for Nanotechnology, Materials Science and Technology of Polymers, \\ University of Twente, P.O. Box 217, 7500 AE Enschede, The Netherlands \\ ${ }^{\mathrm{b}}$ Physikalisches Institut, Wilhelm-Klemm-Str. 10, Westfälische Wilhelms-Universität, D-48149 Münster, Germany
}

Received 14 May 2007; received in revised form 8 June 2007; accepted 8 June 2007

Available online 19 June 2007

\begin{abstract}
The relationship between surface chemistry and morphology of flame treated low-density polyethylene (LDPE) was studied by various characterization techniques across different length scales. The chemical composition of the surface was determined on the micrometer scale by Xray photoelectron spectroscopy (XPS) as well as with time of flight secondary ion mass spectrometry (ToF-SIMS), while surface wettability was obtained through contact angle (CA) measurements on the millimeter scale. The surface concentration of hydroxyl, carbonyl and carboxyl groups, as a function of the "number" of the flame treatment passes (which is proportional to the treatment time) was obtained. Moreover, a correlation was found with chemical composition and polarity, emphasizing the role of oxygen-containing functional groups introduced during the treatment. Carboxyl functional groups were specifically identified by fluorescent labeling and the results were compared with the ToF-SIMS data. In addition, atomic force microscopy (AFM) was used to evaluate changes in surface topography and roughness on the nanometer to micrometer length scales. After flame treatment, water-soluble low molecular weight oxidized materials (LMWOM), which were generated as products of oxidation and chain scission of the LDPE surface, agglomerated into small topographical mounds that were visible in the AFM micrographs. After rinsing the flame treated samples with water and ethanol, bead-like nodular surface structures were observed. The ionization state of flame treated LDPE surfaces was monitored by chemical force microscopy (CFM). The effective surface $\mathrm{p} K_{\mathrm{a}}$ values of carboxylic acid ( $\left.-\mathrm{COOH}\right)$ obtained by AFM were revealed by chemical force titration curves and the effective surface $\mathrm{p} K_{\mathrm{a}}$ values were found to be around 6 .
\end{abstract}

(C) 2007 Elsevier B.V. All rights reserved.

Keywords: Flame treatment; Low-density polyethylene; ToF-SIMS; XPS; AFM; Fluorescence microscopy

\section{Introduction}

Low-density polyethylene (LDPE) has been widely used in various applications owing to its excellent physical and chemical bulk properties. These include light mass, low toxicity, excellent electric insulation, mechanical durability, good chemical resistance and ease of processing and molding $[1,2]$. However, the polymer rarely possesses surface properties that promote successful applications in fields requiring adhesion, printability, and biocompatibility [3]. For instance, the non-polar LDPE surfaces are fairly hydrophobic, i.e. water contact angles around $100^{\circ}$ (advancing) and $70^{\circ}$ (receding) are typically found. It is therefore difficult to bind these

\footnotetext{
* Corresponding author. Tel.: +3153 4892967; fax: +31534893823.

E-mail address: g.j.vancso@tnw.utwente.nl (G.J. Vancso).
}

hydrophobic polymer surfaces directly with other substances, such as adhesives, printing inks, paints, and various metal surfaces. Thus, technologies that involve surface engineering to convert inexpensive LDPE into valuable finished goods have become more important. In order to address these issues, treatments to increase the surface energy of the polymer by introducing polar functionalities are essentially desirable [4-6].

In recent decades, numerous technologies have been extensively developed to modify the surface properties of polymeric materials in order to yield valuable finished products. Examples of such technologies include plasma, chemical, corona, flame, and ion implantation treatments [6-14]. These techniques affect only the chemical and physical properties of the outermost surface layer with an effective skin layer depth depending on the type of treatment and treatment conditions, and thus, they do not alter the bulk properties [13-15]. As is well known, plasma treatment has become increasingly 
important in the last 20 years and it is possible to treat even substrates with complex shapes. The various high energy species present in the plasma induce the formation of free radicals in the polymeric chains and thus permits the formation of certain polar functional groups on the polymeric surface which enhance the surface adhesion. In addition, the treatment is fast, clean, environmentally safe, uniform and precisely controllable [6,13,16-24]. On the other hand, clear disadvantages of the plasma treatment include the fact that it encompasses a complex process and that the adjustment of the plasma discharge parameters constitutes a complicated task $[14,22]$. Moreover, changes in the surface chemistry obtained by plasma treatment are not permanent and this may affect industrial applications [23].

Wet chemical treatments are simple and benign methods for modifying polymer surfaces. Noteworthy studies have focused on changes in surface chemical composition and morphology upon etching with strong acids to convert smooth hydrophobic polymer surfaces to rough hydrophilic ones [25-27]. However, wet chemical treatments involve a number of additional processing steps, such as washing, rinsing and drying. It is rather expensive and gives rise to the environmental problem of disposing of a large amount of toxic waste [28].

For the last 50 years, corona discharges and flame are the most widely used pretreatment methods for polymer films in industry. The corona discharge treatment is generally used for the pretreatment of polyolefin films whereas the flame treatment is used for thicker sections. The corona discharge treatment produces significant changes in the surface topography of polymers, and flame is probably the oldest type of plasma known to humanity. This latter method is extensively used for treating plastic bottles and other moldings [11]. Compared with the corona treatment, flame treatment offers a number of practical advantages, including the ability to achieve extensive surface oxidation and excellent wettability at extremely short processing times. In addition, this method does not generate toxic corrosive ozone and displays no significant loss of treatment upon aging [29]. Though a straightforward set-up is required for this technique, a certain craftsmanship is still needed to obtain consistent results [30]. Currently, the flame treatment is receiving a renewed industrial interest as a technique for modifying films because of major improvements in its safety, reliability and ease of operation [31]. An in depth understanding and a close survey of the physical and chemical properties of flame treated polymer surfaces are required to gain an understanding and be able to carry out an optimization of the process. Unfortunately, available knowledge is currently insufficient.

In the present work, the chemical nature of flame treated LDPE surfaces have been extensively examined by various surface sensitive analytical techniques, including XPS, ToFSIMS, fluorescence microscopy (FM) and CA. In addition, AFM was used to investigate the surface structure and topography of the films on a nanometer resolution before and after treatment. Moreover, the surface ionization state of flame treated LDPE was studied by CFM. The experimental results displayed a multifunctional and responsive surface after flame treatment of LDPE, and should be of help when developing PE commodities with designer surfaces for a variety of applications.

\section{Experimental}

\subsection{Materials}

Additive free LDPE films (with thicknesses of $0.2 \mathrm{~mm}$ ) were obtained from DSM (Geleen, The Netherlands). The melting point and weight percentage of crystallinity, as measured by differential scanning calorimerty (DSC) at a scan rate of $10 \mathrm{~K} /$ min (Perkin-Elmer DSC-7), were $113.5^{\circ} \mathrm{C}$ and $32.1 \%$, respectively. Samples of the films were cut into pieces of ca. $1 \mathrm{~cm}$ $\times 1 \mathrm{~cm}$ prior to treatment. After refluxing in dichloromethane (Merck) for $30 \mathrm{~min}$, the films were rinsed in ethanol (Merck) and dried in a stream of nitrogen immediately prior to use.

\subsection{Flame treatment}

A mixture of natural gas and air were combusted by a nozzle type burner and this procedure constituted the film flame treatment in our experiments. For an efficient treatment, variables such as the air to gas ratio, air and gas flow rates, the distance between the tip of the flame and the object to be treated, as well as the treatment time were controlled [11]. The flow rate of the mixture of natural gas and air was ca. $12.3 \mathrm{~cm}^{3} / \mathrm{s}$ and the equivalence ratio, which is defined as the stoichiometric oxidizer:fuel ratio divided by the actual oxidizer:fuel ratio, was ca. 0.93. A longer exposure to the high temperature flame within one pass caused the films to become easily destroyed and was not very effective for increasing the amount of functional groups. In order to increase the oxidation at the surface, repeated cycles of the flame treatment were carried out. The LDPE films were repeatedly pulled across the tip of the flame at a distance of ca. $0-1 \mathrm{~mm}$ at a speed of ca. $0.5 \mathrm{~m} / \mathrm{s}$. The flame cone exhibited an approximately $1 \mathrm{~cm}$ length, i.e. the exposure time of the film to the flame was about $0.02 \mathrm{~s}$. The frequency of repetitions was defined as the treatment number, which was proportional to the total treatment time. Thus the total treatment time varied from 0 to $4 \mathrm{~s}$. For all experiments, the flame treatment number had to be lower than 200 or else the LDPE films became deformed.

\subsection{Contact angle experiments}

The contact angles were determined by the sessile drop technique using Millipore water and a contact angle microscope (Data Physics, OCA 15plus) at room temperature and ambient humidity. All contact angles were measured on both sides of the drop and the results were averaged. Each contact angle reported in this work was an average of the values obtained for a minimum of three points on the sample surface.

\subsection{X-ray photoelectron spectroscopy (XPS)}

The atomic compositions of the surfaces of the flame treated LDPE samples were analyzed by angle-resolved XPS on a PHI 
Quantum 2000 Scanning ESCA microprobe (Chanhassen, MN) using a $\mathrm{Al} \mathrm{K} \alpha \mathrm{X}$-ray source at a variable take-off angle between $10^{\circ}$ and $60^{\circ}$. A $25 \mathrm{~W}$ X-ray beam with a $100 \mu \mathrm{m}$ diameter scanned over a $700 \mu \mathrm{m} \times 300 \mu \mathrm{m}$ area. Atomic concentrations were determined by numerical integration of the relative peak areas using the Multipak software with supplied sensitivity factors (C 1s: 0.312; O 1s: 0.733) [32].

\subsection{Fluorescence microscopy (FM)}

Fluorescence micrographs were obtained with a Zeiss LSM 510 confocal fluorescence microscope. Images of dry samples on glass cover slips were recorded at room temperature on a Plan-Apochromat ${ }^{\circledR} 63 \times / 1.4$ numerical aperture oil-immersion objective. Fluoresceinamine, excited with the $488 \mathrm{~nm}$ line of an $\mathrm{Ar}^{+}$laser, was used as dye. The fluorescence emission of the dye was recorded with photomultiplier tubes (Hamamatsu R6357) after spectral filtering with a 500-550 nm bandpass filter and a $650 \mathrm{~nm}$ longpass filter. Images with maximum pinhole diameters were acquired.

\subsection{Coupling of fluoresceinamine}

First, untreated and flame treated LDPE films were rinsed thoroughly with water and ethanol. Following this step, the LDPE films were activated for fluorescence studies by immersion for $2 \times 30 \mathrm{~min}$ in aqueous solutions of $1 \mathrm{M} \mathrm{1-}$ ethyl-3-(dimethylamino)-propylcarbodiimide (EDC), and $0.2 \mathrm{M} \mathrm{N}$-hydroxysuccinimide (NHS), respectively. Subsequently, the films were immersed in a fluoresceinamine solution (100 mM, PB buffer, $\mathrm{pH}$ 7.4) for an additional 30 min, after which they were taken out, rinsed with PB buffer and Milli-Q water, and dried in a stream of nitrogen. Fluoresceinamine, EDC and NHS were all purchased from Aldrich and were used as received.

\subsection{Time of flight secondary ion mass spectrometry (ToF- SIMS)}

In ToF-SIMS, a pulsed, focused, energetic ion beam bombards a surface, leading to interactions that cause the emission of positive and negative secondary ions [33,34]. The instrument used was a reflectron type time-of-flight mass spectrometer, with a design equivalent to that of the 'ToF-SIMS IV'. An electron impact ion source $\left(10 \mathrm{keV},{ }^{40} \mathrm{Ar}^{+}\right)$was used for generating primary ion pulses for static ToF-SIMS. The pulsed ion beam was rastered over an area of $200 \mu \mathrm{m} \times 200 \mu \mathrm{m}$. The detected mass range varied from 1 to $3500 \mathrm{amu}$ and the mass resolution $m / \Delta m$ was better than 5000 at mass 41 . For charge compensation, a low energy electron flood gun was utilized.

\subsection{Atomic force microscopy (AFM)}

Surface topography measurements were carried out with a NanoScope IIIa multimode AFM [Veeco Digital Instruments (DI), Santa Barbara]. Tapping mode AFM scans was performed in air using a non-coated silicon tip with a spring constant of
10-20 N/m (Nanosensors, Wetzlar, Germany). Features on the nanometer scale were imaged on a minimum of three different areas on the samples.

AFM force volume imaging was as described in a previously published paper [35]. Force volume images, which give information about laterally resolved surface adherence, were performed in a liquid environment with the AFM controller fitted with a DI liquid cell (volume $\sim 50 \mu \mathrm{L}$ ). Triangular shaped silicon nitride cantilevers and silicon nitride tips (DI) coated with ca. $2 \mathrm{~nm}$ Ti as the adhesion layer and ca. $50 \mathrm{~nm} \mathrm{Au} \mathrm{in} \mathrm{high}$ vacuum were use in these AFM experiments. Functional groups for chemical force microscopy were introduced at the AFM tip surface by modifying gold-coated $\mathrm{Si}_{3} \mathrm{~N}_{4}$ cantilevers with selfassembled monolayers (SAMs). The functionalized tips were prepared by immersion in a $1 \mathrm{mM} 11$-mercapto-1undecanol solution in ethanol during $10-40 \mathrm{~h}$ at room temperature. The so-obtained tips were kept in solution between measurements, rinsed in ethanol and dried in a stream of nitrogen immediately prior to use. The spring constants of the cantilevers ranged from 0.14 to $0.18 \mathrm{~N} / \mathrm{m}$, as obtained by the method of Hutter and Bechhoefer [36] according to the equipartition method which consists of measuring the thermal excitation of the tip to compute its spring constant. The specific details of the CFM procedure were similar to those reported for previous studies [37].

\section{Results and discussion}

\subsection{Surface wettability}

The wetting properties of the LDPE samples on the millimeter length scale were evaluated by contact angle measurements. Fig. 1 shows the variation of the water contact angle with the flame treatment as a function of treatment number. The observed advancing angle was found to change from $100^{\circ}$ for the untreated sample to $47.5^{\circ}$ for the sample treated 200 times. The receding angle changed from $72^{\circ}$ to $12^{\circ}$. This reduction in contact angle demonstrated an increase in the

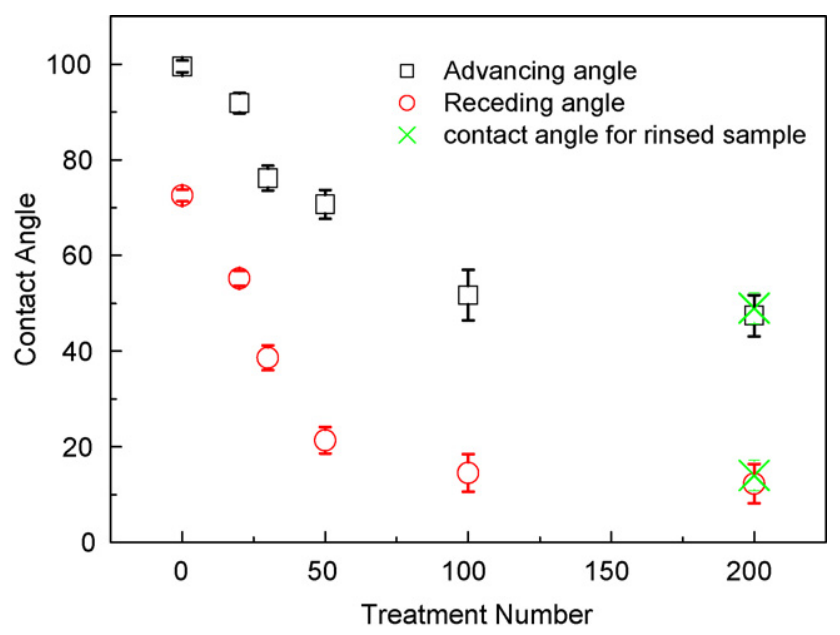

Fig. 1. The variation of surface contact angles measured with $\mathrm{H}_{2} \mathrm{O}$ as a function of the number of passes of flame treatment of LDPE. 
surface wettability and surface energy following the flame treatment, as was expected. The diminution of the water contact angle occurred rapidly at first and then slowed down as the number of flame treatments exceeded 100. This behavior indicated that the amount of polar functional groups at the LDPE surface increased for an increasing number of flame treatments up to 100 after which the surface became saturated. Interestingly, no significant difference was observed between the contact angles of treated and washed samples. The difference between the advancing and receding contact angles, i.e. the contact angle hysteresis, was also found to increase with an increasing number of treatments. It has been widely accepted that contact angles are sensitive to changes in both the surface topography and the chemical behavior [38]. For the flame treated LDPE in the present report, the surface roughness only displayed minor changes on the micrometer scale (as will be shown in the following AFM study). Thus, with respect to the lowering of the water contact angle, the effect of the roughness was expected to be small. Contact angle hysteresis is usually ascribed to a variety of causes including structural disorder, surface roughness and heterogeneity [39]. In the present case, the observed hysteresis may result from the combination of the presence of an increasing amount of polar groups at the surface and their reorientation under influence of the liquid phase. This raises the question regarding what kind of surface functional groups that were introduced by the flame treatment and what the mechanism behind the wettability changes was. In the following section, the atomic percentage of oxygen on treated and washed samples were compared from XPS results to identify the chemical nature of the species introduced through the treatment.

\subsection{Surface chemistry modification}

XPS is utilized to probe the chemical composition and identity of the functional groups that are present within the outermost layer of a film surface. In order to study the in-depth distribution of the chemical composition on the flame treated LDPE films, angle resolved XPS (ARXPS) was used at take-off angles varying from $10^{\circ}$ to $60^{\circ}$. The sampling depth of XPS is related to the relative orientation of the incident $\mathrm{X}$-ray beam and the sample surface. The value of the sampling depth, $l$, for each take-off angle can be calculated according to Eq. (1):

$l=3 \lambda \sin \theta$

where $\lambda$ is the inelastic mean free path of the electrons (ca. $40 \AA$ for C $1 \mathrm{~s}$ from PE) and $\theta$ is the take-off angle [40].

The surface concentration of oxygen in the LDPE films as determined by XPS is shown in Table 1. The untreated LDPE sample was analyzed to determine the cleanliness of the original surface, and it can be noted that the untreated LDPE surface showed less than $2 \%$ oxygen. It was also observed that the oxygen concentration was significantly greater in the treated films than in their untreated counterparts. The oxygen concentration increased with an increasing number of treatments. The surface concentration of oxygen was found to be $10.71 \%$ (take-off angle was $60^{\circ}$ ) whereas $12 \%$ was detected by
Table 1

The oxygen concentration of flame treated LDPE surface vs. the number of treatments as obtained by angle resolved XPS at varying take-off angles

\begin{tabular}{lllllc}
\hline$\theta\left({ }^{\circ}\right)^{\mathrm{a}}$ & $l(\mathrm{~nm})^{\mathrm{b}}$ & $\begin{array}{l}\text { Untreated } \\
\text { LDPE }\end{array}$ & $\begin{array}{l}\text { Treated } \\
100 \text { times }\end{array}$ & $\begin{array}{l}\text { Treated } \\
200 \text { times }\end{array}$ & $\begin{array}{l}\text { Rinsed } \\
\text { sample }\end{array}$ \\
\hline 10 & 2.1 & 1.2 & - & 13.1 & 11.9 \\
20 & 4.1 & 1.3 & - & 11.3 & 9.2 \\
30 & 6.0 & 1.6 & - & 10.8 & 8.3 \\
40 & 7.7 & 1.4 & - & 10.8 & 8.5 \\
45 & 8.5 & 1.0 & 6.7 & 9.6 & 8.0 \\
60 & 10.4 & 1.3 & - & 10.7 & 7.2 \\
\hline
\end{tabular}

${ }^{\text {a }} \theta$ is the take-off angle.

${ }^{\mathrm{b}} l$ is the estimated sampling depth value calculated by Eq. (1).

Briggs et al. in an independent study (take-off angle was $90^{\circ}$ ) [41]. The LDPE film treated 200 times was rinsed for $1 \mathrm{~min}$ in a solution of ethanol and water and this procedure resulted in a drop in the $\mathrm{O}$ concentration as observed by XPS. This was attributed to the presence of low amounts of oxidized, low molar mass fractions of the polymer which were weakly bound to the surface and removed by the solvent [42]. Moreover, for the same flame treated specimen, the $\mathrm{O}$ concentration was found to increase with a decreasing take-off angle, i.e. with decreasing sample depth. For the sample treated 200 times, the oxygen concentration decreased by $\sim 18 \%$ when comparing measurements with sampling depths of $\sim 10.4$ and $\sim 2.1 \mathrm{~nm}$. Although the angle-dependent XPS experiment was a valuable tool to obtain information about the chemical differentiation in the surface layer, care should be taken when interpreting the sampling depth value. Since our LDPE film had a rough surface, it could not be definitively concluded that the flame treatment could modify the LDPE surface any deeper than the XPS sampling depth. Nevertheless, the suggested depth of oxidation was in the range $20-50 \mathrm{~nm}$.

As has been proposed elsewhere $[41,43]$, flame treatment introduces hydroxyl, carbonyl, and carboxyl groups on the surface of PE films. In addition to giving qualitative atomic concentrations on the sample surface, XPS spectra can also provide chemical bonding information. Typical spectra for LDPE surfaces before and after flame treatment are shown in Fig. 2. The $\mathrm{C} 1 \mathrm{~s}$ spectrum of the untreated LDPE contained a symmetric $\mathrm{C}-\mathrm{C}$ peak and no chemical shift was observed for this signal after treatment for 200 times (Fig. 2a). This result indicated the presence of only one valence state for carbon which corresponded to $\mathrm{C}_{x} \mathrm{H}_{y}$ functionalities (i.e. $-\mathrm{CH},-\mathrm{CH}_{2}$, $-\mathrm{CH}_{3}$ ). In contrast, flame modification led to the appearance of a shoulder at higher binding energies, which was taken as an indication of the buildup of oxygenated carbon centers. This result was also consistent with the observed variation in the $\mathrm{O}$ concentration. A simple deconvolution of the $\mathrm{C} 1 \mathrm{~s}$ spectrum for the LDPE treated 200 times demonstrated three peaks at higher binding energies (BE) relative to the primary hydrocarbon peak. These peaks were assigned to alcohol and/or ether $(\mathrm{C}-\mathrm{O})$, as well as carbonyl $(\mathrm{C}=\mathrm{O})$ and carboxyl $(\mathrm{O}-\mathrm{C}=\mathrm{O})$ groups (Fig. 2b). The deconvoluted $\mathrm{O}$ 1s spectra for the flame treated LDPE were not particularly informative due to contributions of every possible chemical environment 

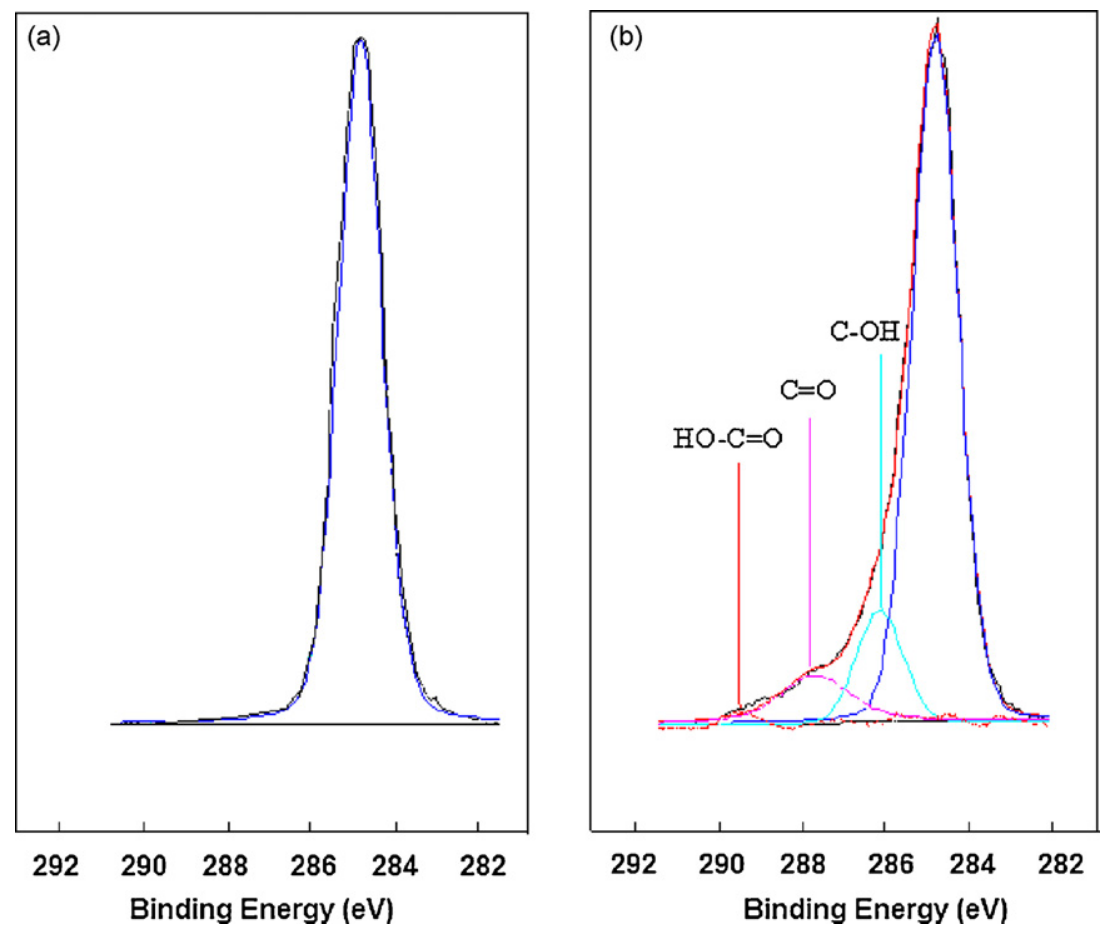

Fig. 2. High-resolution C 1s XPS spectra of (a) untreated LDPE and (b) LDPE treated 200 times.

for $\mathrm{O}$ except $\mathrm{O}-\mathrm{C}=\mathrm{O}$ which has a binding energy some $1.5 \mathrm{eV}$ higher. The relative concentrations of functional groups were obtained by normalizing the integrated peak areas. It was also observed that the relative concentration remained constant with the number of flame treatments; i.e. the ratio among $\mathrm{C}-\mathrm{O}: \mathrm{C}=\mathrm{O}: \mathrm{O}-\mathrm{C}=\mathrm{O}$ groups was found to be 46:44:10.

SIMS has a shallower observation depth than XPS. In other words, the observed fragments are emitted from the first 1-3 top monolayers of the surface, as compared to in the ca. $1-10 \mathrm{~nm}$ layer in XPS analysis. Therefore, ToF-SIMS measurements were also carried out in order to quantitatively study the surface composition of flame treated LDPE as a function of the number of treatments. As mentioned above, by analyzing and deconvolution, the XPS results concerning the $\mathrm{C}$ 1s peak could provide information about the chemical species formed as a result of the surface modification. A direct indication of the composition of these species can be obtained by analyzing the ToF-SIMS data. But the difference in the sampling depths must be kept in mind.

All the treated LDPE films exhibited qualitatively similar ToF-SIMS spectra. The secondary ion intensities of oxygencontaining functional species show an enhancement with the treatment number. Fig. 3 shows typical spectra of untreated LDPE and the LDPE sample treated 200 times at different $\mathrm{m} / \mathrm{z}$ range. Apart from the presence of hydrocarbon peaks, i.e. $\mathrm{C}_{n} \mathrm{H}_{m}{ }^{+}$, due to fragmentation of the polymer, the formation of oxygen-containing functional species was directly observed. Table 2 compares the relative intensities of all the significant oxygen-containing fragments up to $\mathrm{m} / \mathrm{z} 100$ from these positive ion spectra. The intensity of the $\mathrm{C}_{2} \mathrm{H}_{3}{ }^{+}$peak was used as an internal reference for the quantitative treatment of relative peak intensity data as shown in an earlier independent study for a hydrocarbon polymer [44].

It should be kept in mind that for the original LDPE sample a small amount of oxygen was observed. The sample surface of LDPE is a "real world" sample surface. It has been exposed to air and the environment. Thus presence of surface contamination can be anticipated. Because we prepared all samples by the same procedure (except for different flame treatment numbers), the contamination is expected to be similar. We use pure LDPE as background, and all data shown in Table 2 were background corrected to obtain "real" concentration values. In Table 2 , the ions $\mathrm{CHO}_{2}{ }^{+}(\mathrm{m} / \mathrm{z} 45), \mathrm{C}_{2} \mathrm{H}_{4} \mathrm{O}_{2}{ }^{+}(\mathrm{m} / \mathrm{z} 60)$, and $\mathrm{C}_{3} \mathrm{H}_{5} \mathrm{O}_{2}{ }^{+}(\mathrm{m} / \mathrm{z}, 73)$ were candidates for fragments derived from carboxylic acid

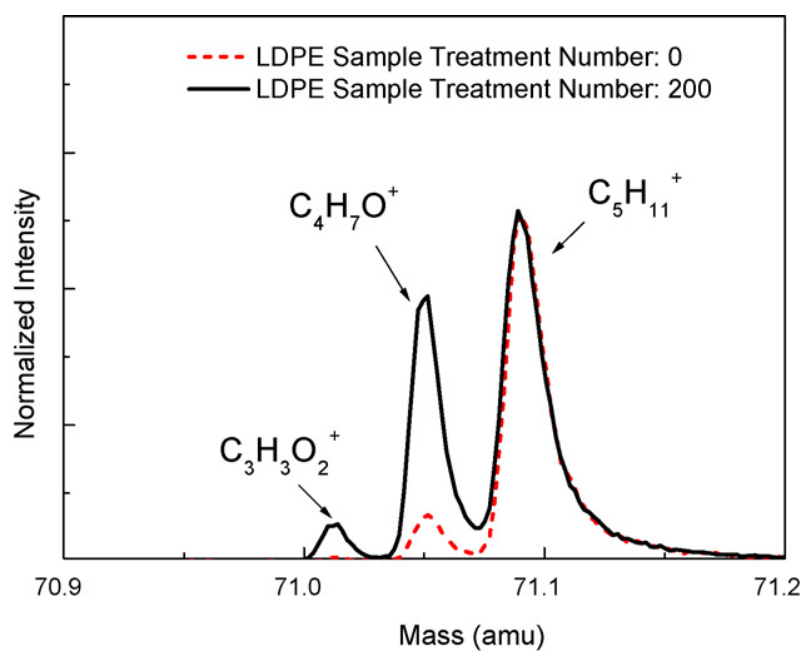

Fig. 3. Positive ToF-SIMS spectrum of untreated and flame treated LDPE surface of fragments at $\mathrm{m} / \mathrm{z}$ around 71 . 
Table 2

The relative intensity $\times 10^{+3}$ of positive ions for flame treated LDPE as obtained by ToF-SIMS using $\mathrm{C}_{2} \mathrm{H}_{3}{ }^{+}$as a standard

\begin{tabular}{|c|c|c|c|c|c|c|c|c|}
\hline Treatment number & $\mathrm{CHO}^{+}$ & $\mathrm{CH}_{3} \mathrm{O}^{+}$ & $\mathrm{C}_{2} \mathrm{H}_{3} \mathrm{O}^{+}$ & $\mathrm{CHO}_{2}^{+}$ & $\mathrm{C}_{2} \mathrm{H}_{5} \mathrm{O}^{+}$ & $\mathrm{C}_{2} \mathrm{H}_{4} \mathrm{O}_{2}^{+}$ & $\mathrm{C}_{4} \mathrm{H}_{7} \mathrm{O}^{+}$ & $\mathrm{C}_{3} \mathrm{H}_{5} \mathrm{O}_{2}^{+}$ \\
\hline 50 & 6.5 & 7.3 & 51.6 & 0.4 & 11.5 & 0.2 & 15.7 & 1.0 \\
\hline 100 & 34.3 & 43.2 & 311.8 & 3.4 & 59.5 & 2.9 & 83.6 & 9.1 \\
\hline 150 & 35.9 & 55.8 & 415.3 & 4.1 & 73.1 & 3.9 & 105.6 & 11.7 \\
\hline 200 & 45.3 & 46.1 & 471.0 & 5.9 & 83.4 & 5.4 & 119.3 & 16.0 \\
\hline
\end{tabular}

functions. Alcohol formed $\mathrm{CH}_{3} \mathrm{O}^{+}(\mathrm{m} / \mathrm{z} 33), \mathrm{C}_{2} \mathrm{H}_{5} \mathrm{O}^{+}(\mathrm{m} / \mathrm{z}$ 45) and carbonyl was from $\mathrm{CHO}^{+}(\mathrm{m} / \mathrm{z}, 29), \mathrm{C}_{2} \mathrm{H}_{3} \mathrm{O}^{+}(\mathrm{m} / \mathrm{z}, 43)$ and $\mathrm{C}_{4} \mathrm{H}_{7} \mathrm{O}^{+}(\mathrm{m} / \mathrm{z}, 71)$. It was observed that the total amount of oxygen-related species increased with the number of treatments. Also the amount of the individual molecular ions increased. The ratio among $\mathrm{C}-\mathrm{O}: \mathrm{C}=\mathrm{O}: \mathrm{O}-\mathrm{C}=\mathrm{O}$ groups was found to be 80:16:4. When compared with XPS, the ToF-SIMS observations displayed a relatively larger concentration of $\mathrm{C}-\mathrm{O}$ fragments. One reason for this difference was presumably the variations in sampling depth indicating the existence of a gradient in the reaction products already for the outermost $10 \mathrm{~nm}$. Finally, when comparing samples treated 50 and 100 times, the concentration of the $\mathrm{O}-\mathrm{C}=\mathrm{O}$ groups increased ca. 10fold while the other two functionalities increased ca. 6-fold. This is an indication of an enhanced formation of $-\mathrm{COOH}$ groups with an increasing number of treatments.

The nature and density of surface functional groups are essential characteristics that affect the properties and applications of a surface, e.g. in surface wetting, and biocompatibility [45]. As discussed above, XPS and ToF-SIMS measurements allowed us to identify and quantify the oxidized functional groups. Often, one of these functional groups is of critical importance, e.g. to provide a specific interaction or to allow attachment of a chemical entity through synthesis. A quantitative characterization of such a specific functional group is clearly crucial and fluorescent labeling offers a possibility in this area. The ease of operation is also important when performing surface analysis. XPS and ToF-SIMS need to be operated in ultrahigh vacuum environments. Fluorescent labeling, on the other hand, is a promising method because of its high sensitivity, ease of operation and in situ applicability. It has been widely used in biological applications [46,47], polymer chemistry [48-51], and study of self assembled monolayers [52-55].
Fluorescent labeling of surface species enables the identification and quantification of very low concentrations of surface functional groups. However, as with any other surface derivatization technique, it provide a lower limit to detect surface coverage [56]. Fluoresceinamine is usually used to label surface aldehyde and carboxyl groups. As was shown in the XPS and SIMS results, carboxyl was the most likely binding group for this dye. In other words, the use of such labeling rendered it possible to selectively detect this functional group. The scheme of fluorescent labeling emphasized in this study is presented in Scheme 1.

Carboxylic acid groups were converted to reactive $\mathrm{N}$ hydroxysuccinimide ester groups by reaction with 1-ethyl-3(dimethylamino)-propylcarbodiimide (EDC) and $N$-hydroxysuccinimide (NHS). Generally, primary amino groups react efficiently with these active esters (also in aqueous media) and yield robust derivatized layers owing to covalent coupling. Thus, the concentration of $-\mathrm{COOH}$ groups could be investigated by fluorescence microscopy using fluoresceinamine as the label. Fig. 4 shows fluorescence microscopy images (image size $200 \mu \mathrm{m} \times 200 \mu \mathrm{m}$ ) of four LDPE samples flame treated a varying number of times and reacted with dye in aqueous medium. The figure displays a clear increase in intensity, and the integrated intensities are also shown in the insets as histogram. For the untreated LDPE film, a fluorescence emission of very low intensity was observed. It can also be noted that the intensity of the fluorescence emission was enhanced with an increasing number of treatments. In addition, a homogeneous fluorescence emission was observed.

An independent confirmation for the increased concentration of carboxylic acid groups was already observed in the SIMS experiment. Fig. 5 shows the normalized $-\mathrm{COOH}$ concentration as a function of treatment frequency as obtained

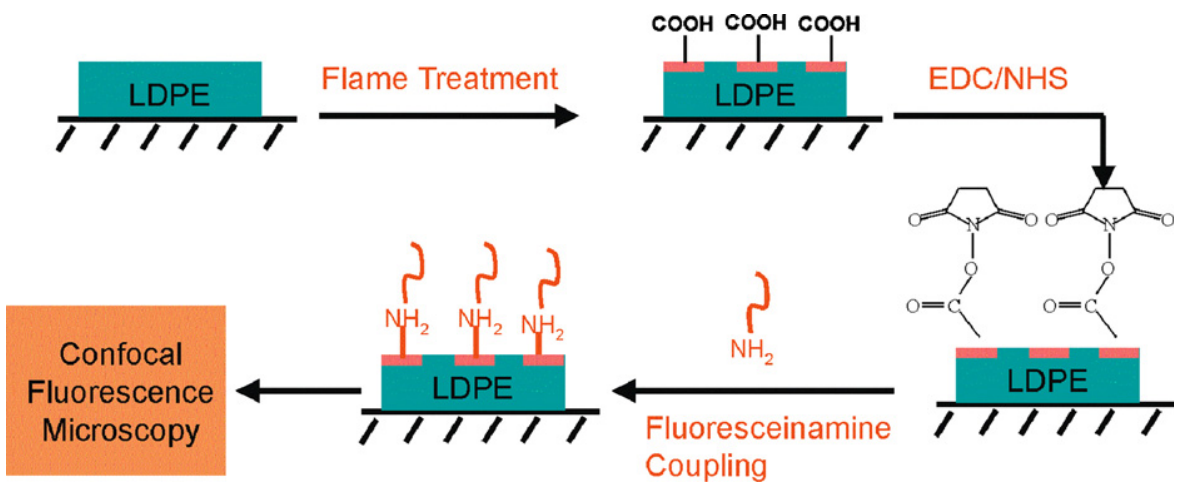

Scheme 1. A schematic portrayal of the immobilization of fluoresceinamine on flame treated LDPE. 

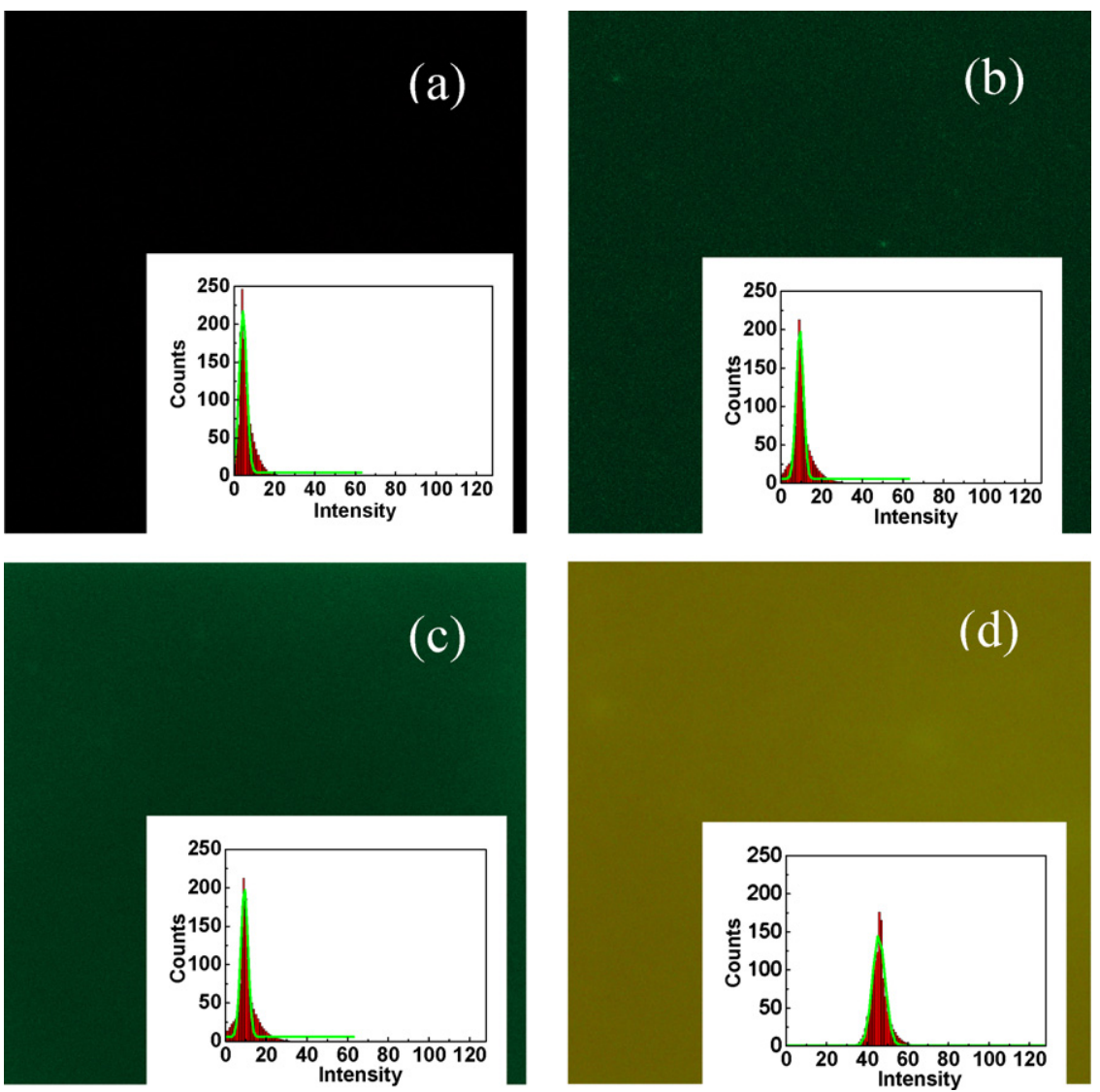

Fig. 4. Fluorescence microscopy images and fluorescence emission intensity histograms of (a) untreated LDPE; (b) LDPE treated 50 times; (c) LDPE treated 100 times; (d) LDPE treated 200 times. Coupling with fluoresceinamine.

by both ToF-SIMS and fluorescent labeling methods. Normalization was performed using the sample treated 200 times as a reference. Both methods gave a nearly linear increase and showed a remarkable quantitative agreement. As for the contact angle measurement, the contact angle reached saturation after 100 treatment cycles. This may be explained by certain of the polar groups reorienting under influence of the liquid phase.

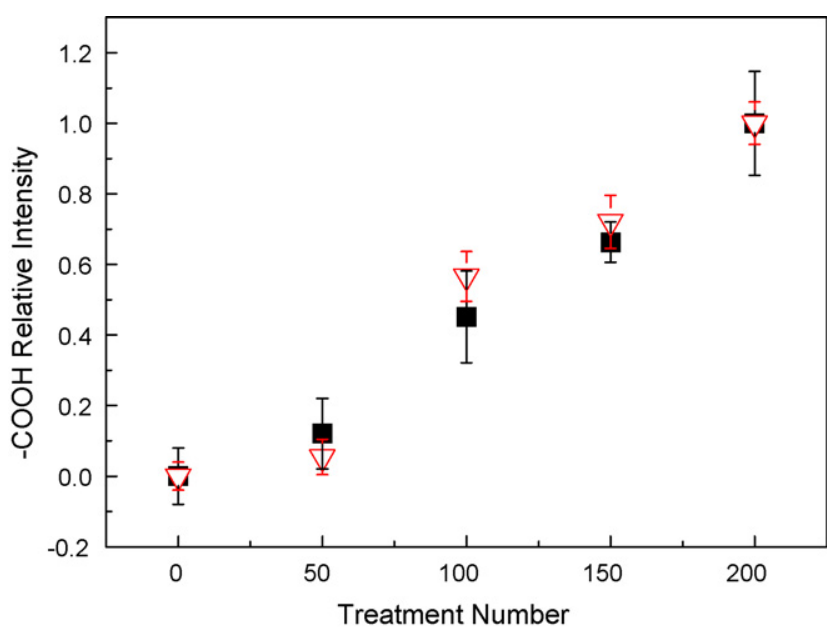

Fig. 5. The normalized [-COOH] of flame treated LDPE as measured by (a) ToF-SIMS $(\nabla)$ and (b) fluorescence microscopy $(\boldsymbol{\square})$ as a function of the number of treatment passes.

\subsection{Nano/micro-surface topography modification}

AFM analysis was carried out in order to observe the topographical changes of flame treated LDPE from the micrometer to the nanometer length scales. Fig. 6 shows representative height images of untreated as well as treated samples. All these images were captured at the scan size of $5 \mu \mathrm{m} \times 5 \mu \mathrm{m}$, with the corresponding $z$ ranges given below the images. For the original untreated LDPE (Fig. 6a), a clearly visible lamellar surface structure was observed, indicating the presence of surface spherulites throughout the film. The lighter areas corresponded to higher regions rendering it possible to observe individual lamellae. After flame treatment of the surface, it became somewhat smoother and exhibited roundshaped features, which formed a nodular structure (Fig. 6b-e). The large nodular features were $0.5-1 \mu \mathrm{m}$ in diameter, and the number of larger features increased for prolonged treatment times. It is known that the original structure of the polymer surface breaks down by some kind of chain scission upon flame treatment [57]. Degradation products and active constituents are thus formed during the treatment. Overney et al. [57] observed that droplet-like structures were formed on polypropylene surfaces at high doses of plasma treatment. These features were identified as low molar mass composition products of the polymer. The large round feature that was apparent in the present case (Fig. 6d and e) resembled the droplets observed by Overney et al., leading us to believe that 

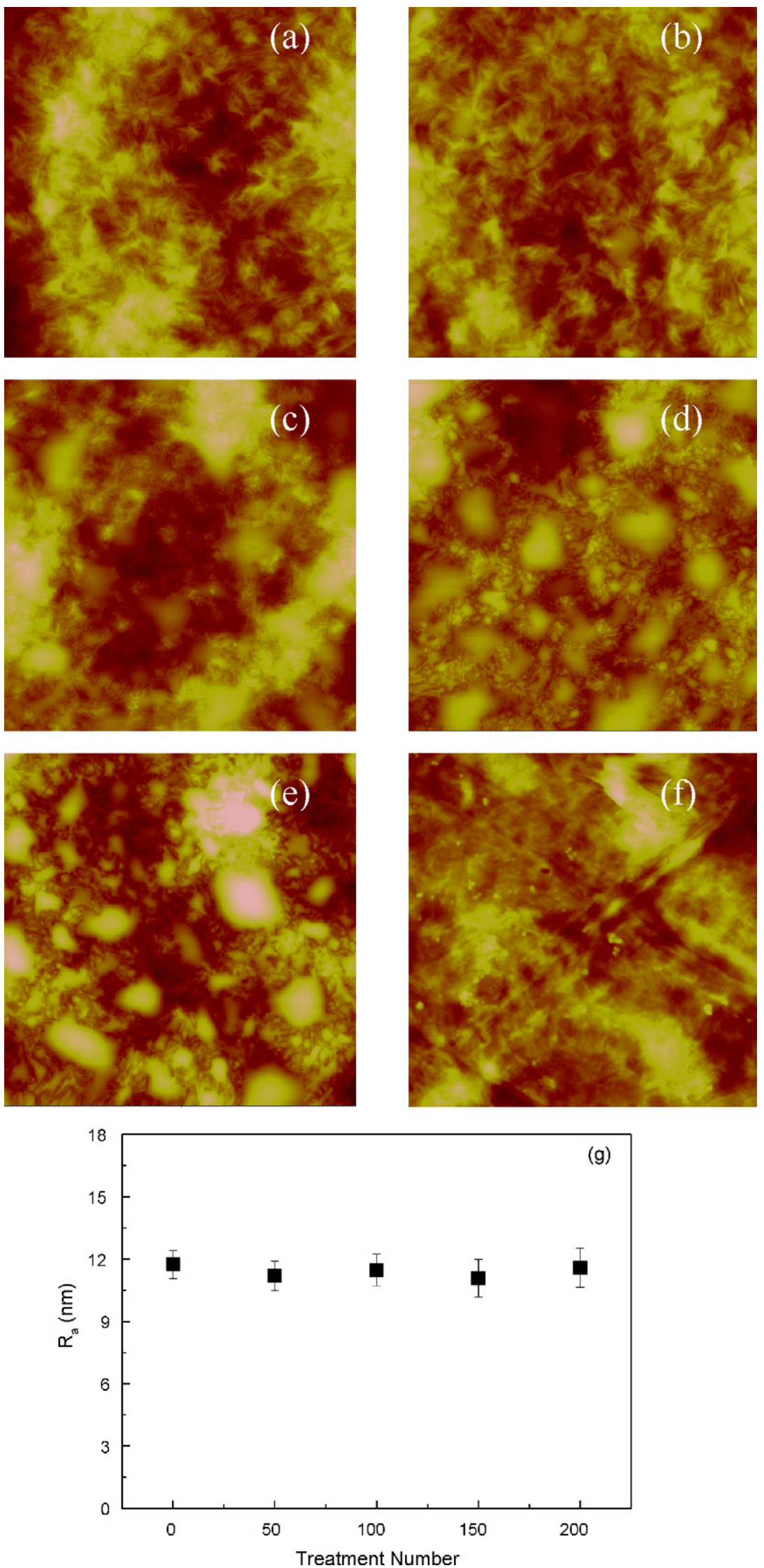

Fig. 6. Tapping mode AFM height images ( $z$-scale $300 \mathrm{~nm}$ ) of (a) untreated LDPE; (b) LDPE treated 5 times; (c) LDPE treated 50 times; (d) LDPE treated 100 times; (e) LDPE treated 200 times; (f) LDPE treated 200 times and subsequently rinsed; (g) the average roughness $R_{\mathrm{a}}$ vs. the number of treatments. The scan size was $5 \mu \mathrm{m} \times 5 \mu \mathrm{m}$ 
escape and rearrangement of degradation products originating from the LDPE surface took place on the treated surface. Fig. $6 f$ shows the topography of a treated sample rinsed with water and ethanol that displayed a different topography. Following the rinsing, neither a mound like structure nor that of a lamella could be observed for the sample treated 200 times. The shape of the globular features (aggregated LMWOM) was explained in terms of the difference in surface energy [58,59]. The LMWOM was easily removed through rinsing with water, due to the fact that it was probably loosely bound to the surface. In addition, it was also found that the rinsed sample displayed small bead-like structures. This means that oxidized polymer would remain at the surface also following rinsing. We propose that corresponding oxidation products of PE were formed in polymer-analogous reactions, by introducing, e.g. carbonyl groups and hydroperoxides [60]. Such products would not be washed off by water treatment $[60,61]$. Fig. $6 \mathrm{~g}$ shows the RMS roughness obtained from AFM images of the sample surface as a function of the flame treatment number. The data was obtained from an average of 3-5 RMS values from a number of AFM images over an area of $5 \mu \mathrm{m} \times 5 \mu \mathrm{m}$. The error bars present the maximum standard deviations. Surprisingly, the RMS roughness did not display a significant change during the flame treatment at the investigated length scale.

\subsection{Surface ionization state}

CFM measurements were carried out by bringing a SAMmodified tip terminated by $-\mathrm{OH}$ entities into contact with the LDPE film and then retracting it. To further investigate the surface ionization state, average pull-off forces in a $500 \mathrm{~nm} \times 500 \mathrm{~nm}$ area were obtained by performing force volume mode scanning on various flame treated samples as a function of the solution $\mathrm{pH}$. The corresponding normalized force titration curves (average pull-off force as a reference of values at $\mathrm{pH} 4$ versus $\mathrm{pH}$ ) on flame treated LDPE are shown in Fig. 7. As was already seen in Fig. $6 \mathrm{~g}$, the mean roughness $\left(R_{\mathrm{a}}\right)$ was not influenced by the flame treatment, and consequently, only a minor influence of the sample topography on the force measurements was expected.

The force titration curves for the flame treated LDPE films exhibited a typical sigmoidal pattern. However, the untreated polymer exhibited a high and almost constant pull-off force of over the entire $\mathrm{pH}$ range investigated. It was suggested that the adherence variation observed by CFM at low $\mathrm{pH}$ values was related to a heterogeneous distribution of protonated carboxyl functional groups $(-\mathrm{COOH})$, as revealed by the ToF-SIMS and XPS experiments. For the flame treated samples, the interactions between the ionizable functional (carboxylic acid) groups and the hydroxyl groups at the tip influenced the $\mathrm{pH}$ dependence of the pull-off force. Since the hydroxyl-terminated functionalized tips did not show any $\mathrm{pH}$ dependence, the titration behavior could be directly attributed to varying degrees of protonation of the $-\mathrm{COOH}$ groups at the LDPE surface. Based on the force titration curves, it was apparent that the strength of adhesion and the extent of attractive interaction diminished with increasing $\mathrm{pH}$.

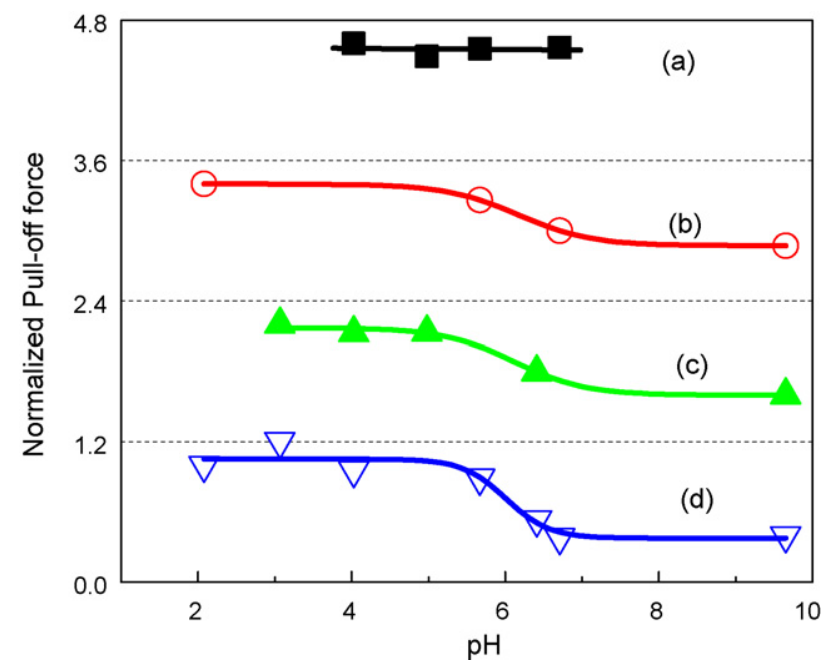

Fig. 7. Normalized chemical force titration curves showing the adhesive force between hydroxyl functionalized tips and flame treated LDPE films as a function of the solution $\mathrm{pH}$. The average pull-off force at $\mathrm{pH} 4$ was set as a reference for each sample. The solid lines have been added as guides to the eye. (a) Untreated LDPE (ם); $\varepsilon=0.27$. (b) LDPE treated 50 times $(\bigcirc) ; \varepsilon=0.44$. (c) LDPE treated 100 times $(\mathbf{\Delta}) ; \varepsilon=0.69$. (d) LDPE treated 200 times $(\nabla)$; $\varepsilon=0.43$. $\varepsilon$ was the average deviation in the adherence as obtained from force volume images.

When the $\mathrm{pH}$ is increased, these protonated functional groups give rise to a force-effective surface $\mathrm{p} K_{\mathrm{a}}$. Such behavior was also observed on the surface-treated LDPE in the present study. The value of the surface $\mathrm{p} K_{\mathrm{a}}$ was evaluated from the force titration curves and was found to be approximately 6 .

Similarly to previous studies [27], it was proposed that for the flame treated LDPE surfaces, the carboxylic acid groups were stabilized by the surrounding methyl groups, leading to the deprotonation occurring at higher $\mathrm{pH}$ values, as compared with the listed homogenous $-\mathrm{COOH}$ solutions $[62,63]$.

\subsection{Oxidation mechanism by flame treatment}

The combustion of hydrocarbons is a highly complex process and many excited species were present in the flame, including free radicals, ions, atoms and electrons [64]. The ion concentration in a hydrocarbon flame is usually low [65], and UV emissions from hydrocarbon flames are primarily due to $\mathrm{OH}$ radicals. Ground state molecular oxygen and long-lived excited oxygen molecules were thought to be non-reactive toward saturated polymers such as PE. Hydrogen abstraction by oxygen molecules is unlikely to occur in a flame. Thus, it was far more likely that the free radicals in the flame were the species responsible for the formation of the polymer radicals $[9,10,30,31,66]$. The mechanism has been unanimously considered to occur by a radical process. In addition, it is known that the chemical reactions evolving in a flame usually proceed through free radical intermediates [67]. The mechanism of surface oxidation by flame treatment likely involves polymer radical formation through abstraction of hydrogen by $\mathrm{O}$ and $\mathrm{OH}$, followed by rapid reaction of the polymer radicals with $\mathrm{O}, \mathrm{OH}$, and $\mathrm{O}_{2}$. The alkoxy, peroxy and hydroperoxy polymer radicals formed by this oxidative attack lead to a wide 
range of oxidized products through further reactions with $\mathrm{O}$ and polymer, or through reactions between the radicals themselves [10,31].

It was found that chain scission $[59,68,69]$ accompanied by oxidative attack leads to the formation of low molecular weight oxidized material which agglomerated into globules at the surface. Such low molecular weight fragments of the polymer at the surface acted as a weak boundary layer. For a larger number of treatments, the degradation was more emphasized. After washing with water, the flame treated LDPE was still oxidized but no longer included scission products with the low molecular weight moieties at the surface. The insoluble oxidized products of flame treatment were probably of high or intermediate molecular weight material.

Degradation of polyethylene is also induced by other external factors including thermo-oxidative processes $[60,61]$. Elevated temperatures can significantly increase the rate of various primary oxidative, and secondary chemical reactions, leading to a decomposition of the polymer. The hydroxyl and carbonyl groups usually account for most of the oxidation products on thermo-oxidative degradation of polyethylene. Such products (formed predominantly in polymer-analogous reactions) would not be washed off by water treatment. In addition, repeated treatment is supposed to gradually increase the $\mathrm{O}$ concentration within the chains which are still bound to the surface increasing concentration of carbonyls, hydroperoxides, etc., not yet broken up to LMWOM. We suggest that this is the reason that from XPS and water contact angle results, the severely treated (200 times) sample shows no large difference compared with the rinsed sample.

\section{Conclusions}

In this paper, the surface of flame treated LDPE was studied by various techniques. A comprehensive view of the effects of the flame treatment at different sampling depths with respect to the sensitivities of the various techniques was obtained. The surface modification of LDPE by this process appeared to be simple and environmentally friendly. Surface physicochemical modification could be attributed to interactions of the polymer with high energy exited species in the flame. These induce modifications characterized by the presence of oxygenated groups at the surface. The introduction of high energy oxidized groups enhanced the LDPE surface energy thus improving its wettability by polar liquids. The number of polar groups showed a constant increase with the number of flame treatments, and the depth of oxidation was also found to be a function of the number of treatments. XPS analysis, using high energy resolution and a refined approach to $C 1$ s curve fitting, provided insights into the quantitative assessment of the type and concentration of functional groups. Positive ToFSIMS spectra were obtained at high mass resolution. The oxygen-containing fragments were identified by accurate mass analysis and subjected to a detailed comparison with XPS results. The dominating functional groups as a result of the flame treatment were identified as hydroxyl, carbonyl and carboxyl groups. The formation of a heterogeneous surface topology exhibiting drop-like features was observed by AFM. The degradation reaction resulted in the formation of low mass by-products via chain scission, which coagulated and appeared as "drops". Washing of the film with water after the flame treatment slightly reduced the atomic percentage of oxygen on the surface and removed the drop-like features. The remaining surface exhibited nodular features in its topography. The surface ionization state was studied by chemical force titration curves. Characteristic surface $\mathrm{p} K_{\mathrm{a}}$ values of approximately 6 were obtained for carboxyl acid groups at the surface. This indicated a nanoscale heterogeneity of the $\mathrm{COOH}$ functions. However, on the microscale and above, the lateral distribution of the functional groups was homogeneous (i.e. beyond the resolution of optical diffraction limit) as evidenced by FM.

\section{Acknowledgements}

The authors gratefully acknowledge financial support from the Dutch Polymer Institute and express their gratitude towards Dr. Holger Schönherr and Dr. Chuanliang Feng for helpful discussions and comments. Finally Dr. Nikodem Tomczak is acknowledged for designing the custom-built Labview application for the AFM force volume image analysis.

\section{References}

[1] R.A. Caldwell, J.E. Woodell, S.P. Ho, S.W. Shalaby, T. Boland, E.M. Langan, M. Laberge, J. Biomed. Mater. Res. 62 (2002) 514.

[2] S. Tajima, K. Komvopoulos, J. Phys. Chem. B 109 (2005) 17623.

[3] M.J. Owen, P.J. Smith, J. Adhes. Sci. Technol. 8 (1994) 1063.

[4] K.S. Kim, C.M. Ryu, C.S. Park, G.S. Sur, C.E. Park, Polymer 44 (2003) 6287.

[5] C.M. Chan, T.M. Ko, Surf. Sci. Rep. 24 (1996) 1.

[6] E.M. Liston, L. Martinu, M.R. Wertheimer, J. Adhes. Sci. Technol. 7 (1993) 1091.

[7] J.S. Chen, Z. Sun, P.S. Guo, Z.B. Zhang, D.Z. Zhu, H.J. Xu, J. Appl. Phys. 93 (2003) 5103.

[8] H. Iwata, A. Kishida, M. Suzuki, Y. Hata, Y. Ikada, J. Polym. Sci. Pol. Chem. 26 (1988) 3309.

[9] F. Severini, L. Di Landro, L. Galfetti, L. Meda, G. Ricca, G. Zenere, Macromol. Symp. 181 (2002) 225.

[10] M. Strobel, V. Jones, C.S. Lyons, M. Ulsh, M.J. Kushner, R. Dorai, M.C. Branch, Plasmas Polym. 8 (2003) 61.

[11] S.M. Desai, R.P. Singh, Adv. Polym. Sci. 169 (2004) 231.

[12] A. Salleo, M.L. Chabinyc, M.S. Yang, R.A. Street, Appl. Phys. Lett. 81 (2002) 4383.

[13] B.K. Kim, K.S. Kim, K. Cho, C.E. Park, J. Adhes. Sci. Technol. 15 (2001) 1805.

[14] C.M. Chan, Polymer Surface Modification and Characterization, Hanser Publishers, Munich, 1994.

[15] D.M. Brewis, D. Briggs, Polymer 22 (1981) 7.

[16] Y. Momose, Y. Tamura, M. Ogino, S. Okazaki, M. Hirayama, J. Vac. Sci. Technol. A 10 (1992) 229.

[17] F. Clouet, M.K. Shi, J. Appl. Polym. Sci. 46 (1992) 1955.

[18] R.H. Hansen, J.V. Pascale, T. De Benedictis, P.M. Rentzepis, J. Polym. Sci. A 3 (1965) 2205.

[19] M. Strobel, S. Corn, C.S. Lyons, G.A. Korba, J. Polym. Sci. Pol. Chem. 23 (1985) 1125.

[20] J.R. Hollahan, A.T. Bell, Techniques and Applications of Plasma Chemistry, John Wiley \& Sons, New York, 1974.

[21] V.N. Vasilets, N.A. Tikhomirov, A.N. Ponomarev, Khim. Vys. Energy 12 (1978) 442. 
[22] B. Olander, A. Wirsen, A.C. Albertsson, J. Appl. Polym. Sci. 91 (2004) 4098.

[23] F. Truica-Marasescu, P. Jedrzejowski, M.R. Wertheimer, Plama Process. Polym. 1 (2004) 153.

[24] C. Klapperich, L. Pruitt, K. Komvopoulos, J. Mater. Sci.: Mater. M 12 (2001) 549.

[25] J.R. Rasmussen, D.E. Bergbreiter, G.M. Whitesides, J. Am. Chem. Soc. 99 (1977) 4746.

[26] J.R. Rasmussen, E.R. Stedronsky, G.M. Whitesides, J. Am. Chem. Soc. 99 (1977) 4736.

[27] H. Schönherr, Z. Hruska, G.J. Vancso, Macromolecules 33 (2000) 4532.

[28] C. Fonseca, J.M. Perena, J.G. Fatou, A. Bello, J. Mater. Sci. 20 (1985) 3283.

[29] M. Strobel, M.C. Branch, M. Ulsh, R.S. Kapaun, S. Kirk, C.S. Lyons, J. Adhes. Sci. Technol. 10 (1996) 515.

[30] F. Garbassi, M. Morra, E. Occhiello, Polymer Surfaces From Physics to Technology, John Wiley \& Sons, Chichester, 1998.

[31] M. Strobel, N. Sullivan, M.C. Branch, J. Park, M. Ulsh, R.S. Kapaun, B. Leys, J. Adhes. Sci. Technol. 14 (2000) 1243.

[32] R.F. Reilman, A. Msezane, S.T. Manson, J. Electron Spectrosc. 8 (1976) 389.

[33] A. Benninghoven, Angew. Chem. Int. Ed. 33 (1994) 1023.

[34] A.M. Leeson, M.R. Alexander, R.D. Short, D. Briggs, M.J. Hearn, Surf. Interf. Anal. 25 (1997) 261.

[35] J. Song, J.F.L. Duval, M.A. Cohen Stuart, H. Hillborg, U. Gunst, H.F. Arlinghaus, G.J. Vancso, Langmuir 23 (2007) 5430.

[36] J.L. Hutter, J. Bechhoefer, Rev. Sci. Instrum. 64 (1993) 1868.

[37] H. Hillborg, N. Tomczak, A. Olah, H. Schönherr, G.J. Vancso, Langmuir 20 (2004) 785.

[38] A.W. Adamson, A.P. Gast, Physical Chemistry of Surfaces, John Wiley \& Sons, New York, 1997.

[39] R.E. Johnson, R.H. Dettre, J. Colloid Interf. Sci. 21 (1966) 610.

[40] Q.T. Le, J.J. Pireaux, R. Caudano, J. Adhes. Sci. Technol. 11 (1997) 735.

[41] D. Briggs, D.M. Brewis, R.H. Dahm, I.W. Fletcher, Surf. Interf. Anal. 35 (2003) 156.

[42] G. Beamson, D. Briggs, High Resolution XPS of Organic Polymers: the Scienta ESCA300 Database, John Wiley \& Sons, Chichester, 1992.

[43] D. Briggs, C.R. Kendall, A.R. Blythe, A.B. Wootton, Polymer 24 (1983) 47
[44] J.C. Vickerman, D. Briggs (Eds.), ToF-SIMS: Surface Analysis by Mass Spectrometry, IMPublications, Chichester, 2001, p. 525.

[45] M.P. Ferraz, J.C. Knowles, I. Olsen, F.J. Monteiro, J.D. Santos, Biomaterials 21 (2000) 813.

[46] R. Benters, C.M. Niemeyer, D. Wohrle, Chembiochem 2 (2001) 686.

[47] E. Apostolova, S. Krumova, N. Tuparev, M.T. Molina, T. Filipova, I. Petkanchin, S.G. Taneva, Colloid. Surf. B 29 (2003) 1.

[48] M. Herold, H. Brunner, G.E.M. Tovar, Macromol. Chem. Phys. 204 (2003) 770 .

[49] K.B. Lee, K.R. Yoon, S.I. Woo, I.S. Choi, J. Pharm. Sci. 92 (2003) 933.

[50] W.L. Wang, Q.G. He, J. Zhai, J.L. Yang, F.L. Bai, Polym. Adv. Technol. 14 (2003) 341.

[51] A. Hollander, Surf. Interf. Anal. 36 (2004) 1023.

[52] T. Ye, E.A. McArthur, E. Borguet, J. Phys. Chem. B 109 (2005) 9927.

[53] S. Flink, F.C.J.M. van Veggel, D.N. Reinhoudt, J. Phys. Org. Chem. 14 (2001) 407.

[54] M. Montalti, L. Prodi, N. Zaccheroni, R. Baxter, G. Teobaldi, F. Zerbetto, Langmuir 19 (2003) 5172.

[55] J. Sagiv, J. Am. Chem. Soc. 102 (1980) 92.

[56] E.A. Mcarthur, T. Ye, J.P. Cross, S. Petoud, E. Borguet, J. Am. Chem. Soc. 126 (2004) 2260.

[57] R.M. Overney, R. Luthi, H. Haefke, J. Frommer, E. Meyer, H.J. Guntherodt, S. Hild, J. Fuhrmann, Appl. Surf. Sci. 64 (1993) 197.

[58] M. Strobel, C. Dunatov, J.M. Strobel, C.S. Lyons, S.J. Perron, M.C. Morgen, J. Adhes. Sci. Technol. 3 (1989) 321.

[59] R.D. Boyd, A.M. Kenwright, J.P.S. Badyal, D. Briggs, Macromolecules 30 (1997) 5429.

[60] F. Gugumus, Polym. Degrad. Stabil. 52 (1996) 131.

[61] P.A. Dilara, D. Briassoulis, J. Agric. Eng. Res. 76 (2000) 309.

[62] C.D. Bain, G.M. Whitesides, Langmuir 5 (1989) 1370.

[63] K. Risch, H. Seitz, Organische Chemie, Schroedel, Hannover, 1981.

[64] A. Gaydon, The Spectroscopy of Flames, Springer, London, 1974.

[65] B. Lewis, G. von Elbe, Combustion, Flames and Explosions of Gases, Academic Press, Orlando, 1987.

[66] E. Sheng, I. Sutherland, D.M. Brewis, R.J. Heath, R.H. Bradley, J. Mater. Chem. 4 (1994) 487.

[67] D. Briggs, C.R. Kendall, Polymer 20 (1979) 1053.

[68] W.R. Penney, K.J. Bell, Ind. Eng. Chem. 59 (1967) 40.

[69] H.K. Yun, K. Cho, J.K. Kim, C.E. Park, S.M. Sim, S.Y. Oh, J.M. Park, J. Adhes. Sci. Technol. 11 (1997) 95. 